\title{
Evaluation of Corrosion Potential Measurements as a Means To Monitor the Storage and Stabilization Processes of Archaeological Copper-Based Artifacts
}

\author{
Karen Leyssens, ${ }^{\dagger}$ Annemie Adriaens, ${ }^{*,+}$ Christian Degrigny, ${ }^{\ddagger}$ and Emmanuel Pantos $\$$ \\ Department of Analytical Chemistry, Ghent University, Krijgslaan 281-S12, B-9000 Ghent, Belgium, \\ Heritage Malta-Conservation Division, East Wing, Royal Naval Hospital, Bighi, Kalkara, Malta, and \\ Daresbury Laboratory, CCLRC, Warrington WA4 4AD, U.K.
}

The focus of this study consists of examining how corrosion potential measurements can contribute in providing information on the effectiveness of storage and stabilization treatments of copper alloys in aqueous solutions. We report on the electrochemical behavior of artificial copper alloy coupons (covered or not with corrosion layers), simulating the behavior of real artifacts, immersed in sodium sesquicarbonate solutions. Particular attention is given to the transformation of the corrosion layer as a function of time. In addition, synchrotron radiation X-ray diffraction measurements are performed before and after the treatment in order to understand the reactions that take place during the immersion processes.

Copper alloys recovered from marine environments can be heavily contaminated with chloride ions. While cuprous chloride (nantokite) may lie dormant in the existing corrosion layer, a reaction with moisture and oxygen causes this unstable compound to expand in volume on conversion to one of the copper trihydroxychlorides (atacamite, paratacamite). This creates physical stress within the object affected, resulting in cracking or fragmentation. Ultimately this behavior, often called bronze disease, can reduce an apparently solid object into a heap of powder. For this reason, archaeological copper alloys recovered from wet saline environments should not be exposed directly to the atmosphere, as the metal will then corrode at an accelerated rate in the oxygen-rich air. ${ }^{1-3}$ Alternatively, the contamination will have to be treated if the copper alloys are to be stabilized against continuing corrosion.

Copper-based objects are therefore usually stored and stabilized (i.e., chloride ions are being leached from the corrosion layer) in a solution using either tap water or diluted sodium sesquicarbonate (equimolar mixture of sodium hydrogen carbonate and carbonate)., ${ }^{2,-6}$ Several other methods for stabilizing

* To whom correspondence should be addressed. E-mail: annemie.adriaens@ ugent.be. Tel: +32926448 26. Fax: +3292644960.

$\dagger$ Ghent University.

‡ Heritage Malta-Conservation Division.

$\S$ CCLRC.

(1) Pearson C. Conservation of Marine Archaeological Objects; Butterworth \& Co. Ltd.: London, 1987.

(2) Scott, D. A. Copper and Bronze in Art: Corrosion, Colorants, Conservation; The Getty Conservation Institute: Los Angeles, 2002. corroded copper alloys are in practice as well, such as washing the objects in alkaline dithionite, in citric acid inhibited with thiourea, or in aqueous acetonitrile; however, the use of tap water or a sodium sesquicarbonate solution remains the most commonly used, since this procedure proves to be the least damaging to the patina. ${ }^{2,5}$ Nevertheless, results often show a certain instability of the artifacts in solution, such as the chemical transformation of the natural patina. Examples include the formation of tenorite $(\mathrm{CuO})^{7}$ or chalconatronite $\left(\mathrm{Na}_{2} \mathrm{Cu}\left(\mathrm{CuO}_{3}\right)_{2} \cdot 3 \mathrm{H}_{2} \mathrm{O}\right) .{ }^{8}$ The occurrence of these side effects means that monitoring of the storage and stabilization treatments remains necessary.

A literature search shows that storage and stabilization processes are mainly monitored by analyzing the chloride concentration of the solution at regular time intervals..$^{5-6,9}$ This is very useful to monitor the chloride removal from the corrosion layer but does not provide information with regard to possible side effects taking place at the metal surface, such as the modification of the natural patina or the development of active corrosion. Therefore, there is still a need for another method, which not only monitors the progression of the stabilization process but also makes sure whether other (unwanted) reactions take place on the metal surface.

In this paper, we evaluate whether monitoring the corrosion potential $\left(E_{\text {corr }}\right)$ as a function of time can be used as an early warning system to detect alterations of copper-based alloys during their storage and stabilization processes. The latter is a relatively simple concept (and therefore easy to use in museums and conservation laboratories), in which the open circuit potential of the object is measured relative to a reference electrode. The

(3) Hamilton, D. L. Methods of Conserving Underwater Archaeological Material Culture, Conservation Files: ANTH 605, Conservation of Cultural Resources I. Nautical Archaeology Program, Texas A\&M University, World Wide Web, http://nautarch.tamu.edu/class/ANTH605, 1998.

(4) MacLeod, I. D. In Proceedings of the 8th triennial ICOM-CC meeting; K. Grimstad, K., Ed.; The Getty Conservation Institute: Sydney, 1987; pp 10791085.

(5) MacLeod, I. D. Stud. Conserv. 1987, 32, 25-40.

(6) Oddy, W. A.; Hughes, M. J. Stud. Conserv. 1970, 15, 183-189.

(7) Pollard, A. M.; Thomas, R. G.; Williams, P. A. Stud. Conserv. 1990, 35, $148-152$.

(8) Horie, C. V.; Vint, J. A. Stud. Conserv. 1982, 27, 185-186.

(9) North, N. A. In Conservation of Marine Archaeological Objects; Pearson, C., Ed.; Butterworth \& Co. Ltd.: London, 1987; pp 207-252. 
Table 1. Composition of the Copper-Based Alloys $(\mathrm{g} / \mathrm{kg})$ (Certified for the Alloying Elements) ${ }^{12}$

\begin{tabular}{cccccc} 
& \multicolumn{2}{c}{$\begin{array}{c}\text { tin bronze } \\
(\mathrm{Cu}-\mathrm{Sn})\end{array}$} & & \multicolumn{2}{c}{$\begin{array}{c}\text { lead bronze } \\
(\mathrm{Cu}-\mathrm{Sn}-\mathrm{Pb})\end{array}$} \\
\cline { 2 - 3 } \cline { 5 - 6 } element & concn & uncertainty & & concn & uncertainty \\
$\mathrm{Sn}$ & 70 & \pm 6 & & 101 & \pm 8 \\
$\mathrm{~Pb}$ & 2.04 & \pm 0.18 & & 92 & \pm 17 \\
$\mathrm{As}$ & 1.94 & \pm 0.20 & & 2.85 & \pm 0.22 \\
$\mathrm{Zn}$ & 1.57 & \pm 0.25 & & 1.48 & \pm 0.24 \\
\hline
\end{tabular}

hypothesis behind the method is that if the corrosion potential does not change as a function of time, the surface composition should be stable. A change of the corrosion potential, on the other hand, could indicate a transformation of the natural patina or the development of active corrosion.

This work focuses on the behavior of single patina layers on copper alloys to evaluate the effect of the treatment solution on different types of corrosion products. The behavior of corroded copper was the subject of previous papers..$^{10,11}$ This paper reports on $E_{\text {corr }}$ versus time plots of a copper-tin and a copper-tin-lead alloy (covered or not with corrosion layers) immersed in a sodium sesquicarbonate solution. Synchrotron radiation XRD (SR-XRD) measurements were performed in parallel to understand the reactions that take place during the immersion processes.

\section{EXPERIMENTAL SECTION}

Materials and Sample Preparation. Because of the uniqueness of the artifacts with which conservators are dealing, it was not advisable to use authentic artifacts for this evaluation study. Instead, commercially available copper alloys were artificially corroded and then studied. The main composition of the copper-tin and copper-tin-lead alloy used in this study is given in Table 1 . The choice of these alloys resulted from a previously performed project, which had the goal to produce and certify a set of copper alloys having a composition representative of typical archaeological copper alloys in Western Europe, covering three periods: the Bronze Age, the Iron Age, and Roman times. ${ }^{12}$ Figure 1 shows an optical micrograph of the two alloys. For a detailed description of the microstructure, the authors refer to a paper by Constantinides et al. ${ }^{13}$

Disk electrodes of 6 -mm diameter were produced using the above-mentioned materials by embedding the pieces of metal in an epoxy resin and ensuring an electrical connection. Prior to corrosion simulation, the electrodes were prepared according to standard metallurgical procedures, which included grinding to 1200-grit SiC paper and polishing on cloth covered with $1-\mu \mathrm{m}$ alumina powder. To remove any adherent $\mathrm{Al}_{2} \mathrm{O}_{3}$ particles, the

(10) Leyssens, K.; Adriaens A.; Degrigny, C. In Proceeding of Metals 2004; Ashton, J., Hallam, D., Eds.; National Museum of Australia Press: Canberra, 2004; pp 332-343.

(11) Leyssens, K.; Adriaens, A.; Dowsett, M. G.; Schotte, B.; Oloff, I.; Pantos, E.; Bell, A. M. T.; Thompson, S. P. Electrochem. Commun. 2005, 7, 12651270 .

(12) Ingelbrecht, C.; Adriaens, A.; Maier, E. A. Certification of Arsenic, Lead, Tin and Zinc (Mass Fractions) in Five Copper Alloys; CRM 691. EUR 19778/1, Office for Official Publications of the European Communities, Luxembourg, 2001.

(13) Constantinides, I.; Gritsch, M.; Adriaens, A.; Hutter, H.; Adams, F. Anal. Chim. Acta 2001, 440, 189-198. surfaces were rinsed thoroughly with deionized water and cleaned in an ultrasonic bath. Afterward, several corrosion protocols, obtained from the literature, were applied to obtain different corrosion products. Six corrosion products commonly found on real artifacts were considered.

Cuprite $\left(\mathrm{Cu}_{2} \mathrm{O}\right)$ is regularly found on copper artifacts and is a stable corrosion product.2,14 To obtain a cuprite layer, the copperbased samples were polarized anodically at $-360 \mathrm{mV}$ (versus a saturated mercury sulfate reference electrode) for $16 \mathrm{~h}$ in a 0.1 $\mathrm{M} \mathrm{Na}_{2} \mathrm{SO}_{4}$ solution (Fluka). ${ }^{15}$

Among the copper chlorides, nantokite $(\mathrm{CuCl})$, atacamite $\left(\mathrm{Cu}_{2}(\mathrm{OH})_{3} \mathrm{Cl}\right)$, and a mixture of atacamite and paratacamite (both isomers of $\mathrm{Cu}_{2}(\mathrm{OH})_{3} \mathrm{Cl}$ ) were selected. Nantokite is considered as the main catalytic agent for active corrosion. The presence of this cuprous chloride as a corrosion product adjacent to the metallic surface can create long-term problems with regard to the stability of an object. In fact, bronze disease or pitting corrosion is usually attributed to this corrosion product. ${ }^{2}$ Samples covered with nantokite were obtained by immersing the samples for $1 \mathrm{~h}$ in a saturated $\mathrm{CuCl}_{2} \cdot 2 \mathrm{H}_{2} \mathrm{O}$ solution (VWR International). After rinsing with deionized water, they were exposed to the atmosphere overnight. ${ }^{16}$

Atacamite and paratacamite are two other important chlorides in bronze corrosion. They are often considered as end products and are formed on top of the active corrosion areas. Atacamite is the most common of the $\mathrm{Cu}_{2}(\mathrm{OH})_{3} \mathrm{Cl}$ isomers but often alters into paratacamite. ${ }^{2,14}$ For the atacamite corrosion, a solution of 15.07 $\mathrm{g}$ of $\left(\mathrm{NH}_{4}\right)_{2} \mathrm{CO}_{3} \cdot \mathrm{NH}_{3}$ (Fluka) and $10.02 \mathrm{~g}$ of $\mathrm{NH}_{4} \mathrm{Cl}$ (Aldrich) in $100 \mathrm{~mL}$ of deionized water was prepared. The copper-based alloys were wetted twice a day with this solution. This procedure was repeated during 5 days. Between each application, the samples were left to dry to the air. After the period of 5 days, the samples were left in the air for another 5 days without any treatment. ${ }^{16}$

-The protocol used to obtain a mixture of atacamite and paratacamite (both isomers of $\mathrm{Cu}_{2}(\mathrm{OH})_{3} \mathrm{Cl}$ ) differed only in the type of solution used: a solution of $10.02 \mathrm{~g}$ of $\mathrm{Cu}\left(\mathrm{NO}_{3}\right)_{2} \cdot 3 \mathrm{H}_{2} \mathrm{O}$ (VWR International) and $10.01 \mathrm{~g}$ of $\mathrm{NaCl}$ (Fluka) in $100 \mathrm{~mL}$ of deionized water was used. ${ }^{16}$

Chalcocite $\left(\mathrm{Cu}_{2} \mathrm{~S}\right)$ is typical for marine artifacts found in anaerobic environments. ${ }^{2}$ The protocol to form chalcocite included placing the samples in a closed box for 30 min together with a mixture of $4 \mathrm{~mL}$ of $20 \% \mathrm{NH}_{4} \mathrm{~S}$ (VWR International) and $20 \mathrm{~mL}$ of deionized water. ${ }^{16}$

The copper sulfates are important primarily as corrosion products of copper alloys exposed to polluted atmospheres in urban environments. ${ }^{2}$ To obtain copper-based alloys covered with brochantite, the samples are immersed 3 weeks in a solution of $6 \mathrm{~g}$ of $\mathrm{KClO}_{3}$ (Aldrich), $3 \mathrm{~g}$ of $\mathrm{CuSO}_{4} \cdot 5 \mathrm{H}_{2} \mathrm{O}$ (Vel) and $4 \mathrm{~g}$ of $\mathrm{Na}_{2} \mathrm{SO}_{4} \cdot 10 \mathrm{H}_{2} \mathrm{O}$ (Fluka) in $1 \mathrm{~L}$ of deionized water adjusted to $\mathrm{pH}$ 3 with $\mathrm{H}_{2} \mathrm{SO}_{4} \cdot{ }^{17}$

Various concentrations of sodium sesquicarbonate solutions are used by conservators for the stabilization of bronze artifacts. Solutions of 5-10 wt \% sesquicarbonate are very common,

(14) De Ryck, I. Chemical characterization of ancient artifacts: Application of microbeam methods, University of Antwerp: Antwerp, 2003.

(15) Beldjoudi, T. Unpublished Rapport Technol. STEP/EDF, Synthèse des Travaux, Valectra, 1999.

(16) Lamy, C. unpublished Rapport du Stage, Université de Nantes ISITEM, 1997.

(17) Hayez, V.; Costa, V.; Guillaume, J.; Terryn, H.; Hubin, A. Analyst 2005, $150,550-556$ 


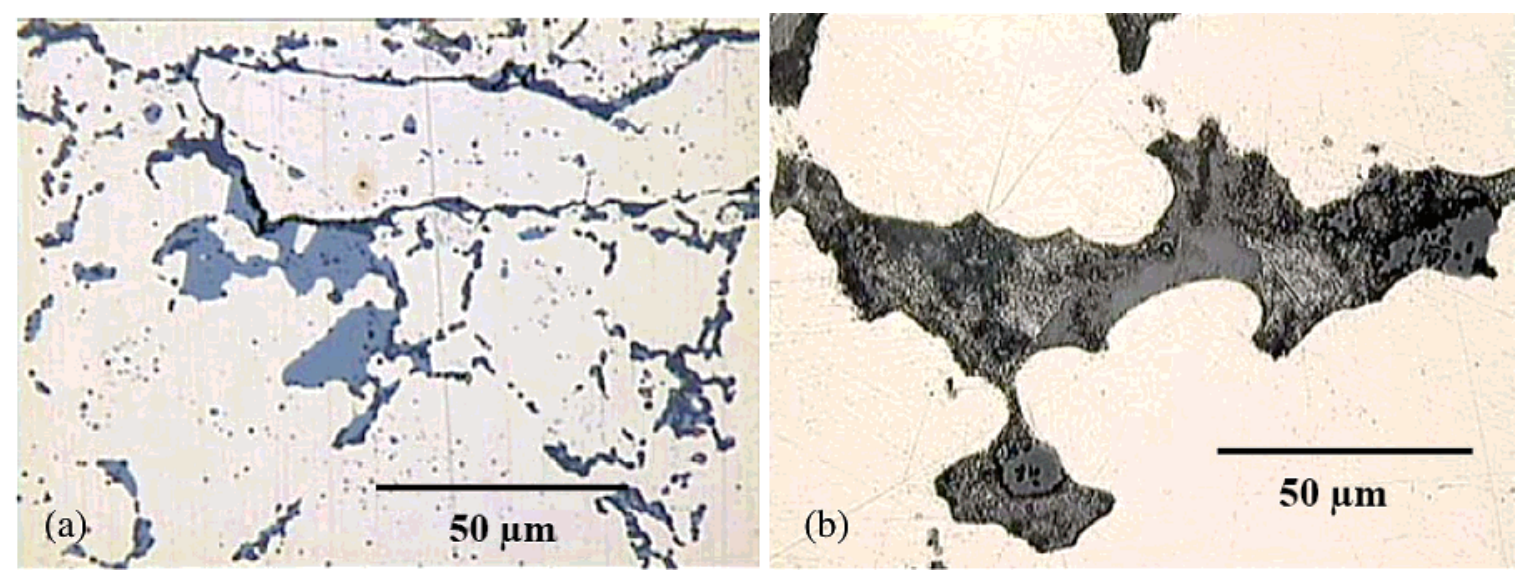

Figure 1. (a) Tin bronze under plane-polarized light (magnification $\times 400$ ). (b) Lead bronze under unpolarized light (magnification $\times 400)$.

but due to side effects, such as the formation of chalconatronite $\left(\mathrm{Na}_{2} \mathrm{Cu}\left(\mathrm{CuO}_{3}\right)_{2} \cdot 3 \mathrm{H}_{2} \mathrm{O}\right)$, lower concentrations are now used. ${ }^{2-5}$ For this study, a $1 \mathrm{wt} \%$ sodium sesquicarbonate solution was prepared by dissolving $11.89 \mathrm{~g} / \mathrm{L} \mathrm{Na}_{2} \mathrm{CO}_{3} \cdot \mathrm{NaHCO}_{3} \cdot 2 \mathrm{H}_{2} \mathrm{O}$ (Sigma) in deionized water ( $\mathrm{pH} 10)$.

Techniques. Corrosion potential measurements were performed using a PC-controlled potentiostat and software package type GPES4.9 (Autolab PGSTAT10, Eco Chemie). A mercury sulfate electrode $\left(\mathrm{Hg} / \mathrm{Hg}_{2} \mathrm{SO}_{4}, \mathrm{~K}_{2} \mathrm{SO}_{4}(\right.$ satd) $)$ was used as reference electrode ( $=640 \mathrm{mV}$ versus standard hydrogen electrode). All potentials in the text are referred to the standard hydrogen electrode (SHE). The disk electrodes were immersed in $150 \mathrm{~mL}$ of the electrolyte solution (a $1 \mathrm{wt} \%$ sodium sesquicarbonate solution). It was decided not to stir the solution during the measurements in order to be able to make a comparison between tap water (used as a storage solution) and sodium sesquicarbonate solution in a later stage.

SR-XRD measurements were performed to determine the surface composition of the electrodes before and after immersion experiments. Powder samples were prepared by scraping the corrosion products off the sample surface. The powder samples were deposited on tape stretched over thin metal sample holders, which were then mounted on the goniometer. Exposure times of $30 \mathrm{~s}$ were used in multibunch $(250 \mathrm{~mA})$ mode. $2 \mathrm{D}$ diffraction patterns were acquired using a CCD detector at station 9.6 of the synchrotron radiation source at Daresbury Laboratory (U.K.). The data were polar-transformed and azimuth-integrated using the ESRF program FIT2D. ${ }^{18}$

\section{RESULTS}

Copper-Tin Alloy. A set of artificially corroded copper-tin coupons was prepared according to the above-mentioned corrosion protocols. The obtained corrosion products were in first instance verified using SR-XRD. In contrast to the corrosion of pure copper where only the desired corrosion product was obtained for each of the protocols, ${ }^{10}$ several side products were formed when corroding the $\mathrm{Cu}-\mathrm{Sn}$ alloy (Table 2). The protocol to obtain cuprite, for instance, also generated cassiterite $\left(\mathrm{SnO}_{2}\right)$, while the nantokite protocol also produced atacamite and paratacamite on the sample. Similar results were obtained when applying the brochantite protocol, which generated additional cuprite.

The corroded copper-tin coupons were immersed in a $1 \mathrm{wt} \%$ sodium sesquicarbonate solution while corrosion potential mea- surements were performed. Measurements were performed at least three times and showed for a particular corrosion product slight differences in time scale, which is most likely due to a variation in the thickness of the corrosion layer. The general behavior, however, resembled each other well. In Figure 2, a set of representative $E_{\text {corr }}$ measurements for the various corrosion products is shown for an immersion period of 14 days. In what follows, the $E_{\text {corr }}$ data for each corrosion protocol will be discussed in more detail and will be related to the obtained SR-XRD measurements.

Both the uncorroded sample and the one corroded with cuprite reach a stable corrosion potential after only a few hours. This corrosion potential is maintained during the rest of the immersion time. According to our hypothesis, this behavior indicates that no significant transformations take place on the surface of these samples. SR-XRD measurements recorded after 14 days of immersion confirm the qualitative composition of the corrosion layers remained the same (Table 2 ).

The corrosion potential of the copper-tin sample covered with nantokite shows on the other hand a lot of variation at the beginning of the immersion, but after a few days, the corrosion potential reaches the same value as obtained for the uncorroded sample and the sample covered with cuprite. SR-XRD data were taken after $1^{19}$ and 14 days (Table 2) of immersion and give the same result, namely, the formation of cuprite and the disappearance of nantokite. The latter suggests that changes of the corrosion layer take place the very first hour to days.

The corrosion potential of the copper-tin sample covered with atacamite strongly increases during the first day, showing afterward a long slow descent in its value. SR-XRD spectra after 14 days (Table 2) show the formation of cuprite. In one case, malachite and cassiterite were formed as well. Atacamite in general does not seem to transform; instead, cuprite is formed in addition. The strong increase in the corrosion potential possibly indicates the formation of a new corrosion product, cuprite in this case, where the slow descent of the corrosion potential with time could be due to the growth of this component.

(18) Hammersley, A. P.; Svensson, O.; Hanfland, M.; Fitch, A. N.; Hausermann, D. High-Pressure Res. 1996, 4, 235-248.

(19) Leyssens, K.; Adriaens, A.; Pantos, E.; Degrigny, C. In Proceedings of the 8th International Conference on Non-Destructive Investigations and $\mathrm{Mi}$ croanalysis for the Diagnostics and Conservation of the Cultural and Environmental Heritage; Parisi C., Buzzanca, G., Paradisi. A., Eds.; Italian Society of Non Destructive Testing Monitoring Diagnostics: Rome, 2005; p 77. 


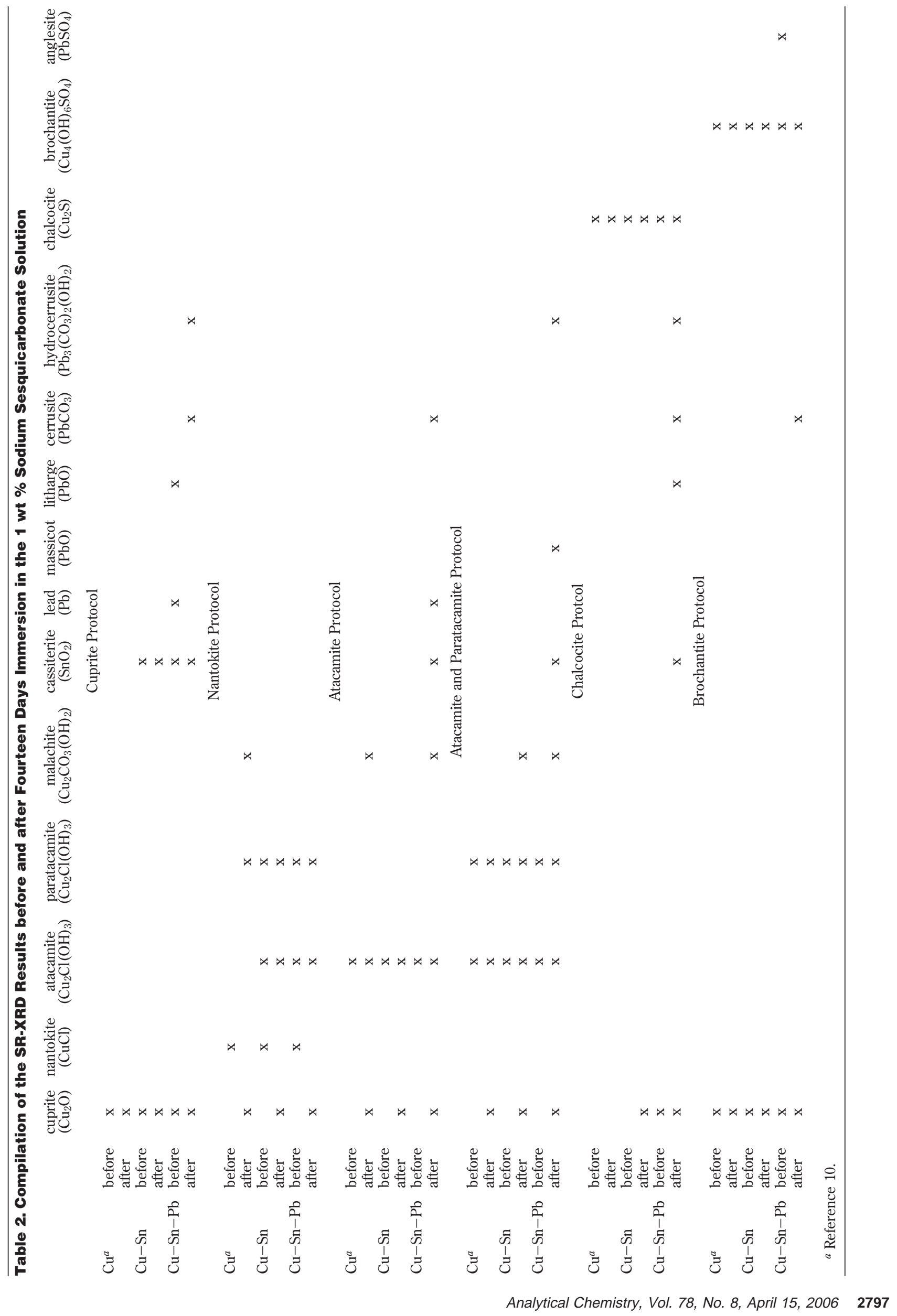




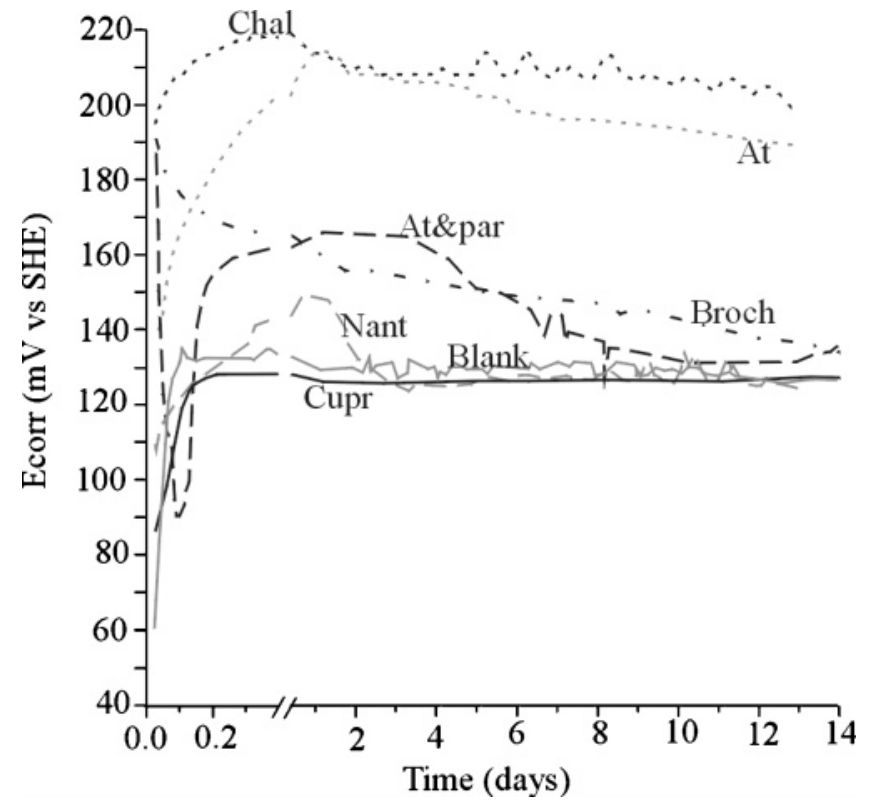

Figure 2. Corrosion potential versus time measurements for a copper-tin alloy covered with different corrosion products and immersed in a $1 \mathrm{wt} \%$ sodium sesquicarbonate solution (blank, uncorroded copper-tin alloy; cupr, copper-tin alloy covered with cuprite; nant, copper-tin alloy covered with nantokite; at, copper-tin alloy covered with atacamite; at\&par, copper-tin alloy covered with a mixture of atacamite and paratacamite; chal, copper-tin covered with chalcocite; and broch, copper-tin alloy covered with brochantite).

The copper-tin alloy sample covered with a mixture of atacamite and paratacamite shows right after its immersion a strong descent in the corrosion potential, after which its value strongly increases again to a maximum, where it stays for a few days (two or three). Later on, the corrosion potential starts to descend slowly to a more or less stable corrosion potential just above the range of copper-tin covered with cuprite. A few of the samples show another increase in the corrosion potential toward the end of 14 immersion days. The SR-XRD spectra (Table 2) show the formation of cuprite, although atacamite and paratacamite remained present on the sample. In some cases, malachite was formed as well. The variation of the corrosion potential during the first few days is most probably caused by the formation of cuprite. This is strongly indicated by the fact that the corrosion potential varies to the corrosion potential of the copper-tin alloy covered with cuprite. Possibly part of the atacamite and paratacamite is reacting as well, which would explain the strong variations in the first hours of the immersion. The small increase of the corrosion potential at the end of the 14 days immersion is probably due to the formation of malachite, since malachite was never found after 1 day of immersion, ${ }^{19}$ and only in some cases after 14 days of immersion.

The copper-tin surface covered with chalcocite shows the same behavior as the copper-tin covered with atacamite in that the corrosion potential strongly increases in the beginning, after which the corrosion potential starts a long slow descent. Also in this case, the SR-XRD spectra (Table 2) show the formation of cuprite. The latter indicates that chalcocite does not transform into other corrosion products; however, (more) cuprite is formed in addition. Therefore, the strong increase of the corrosion potential is most likely due to the formation of cuprite. The higher

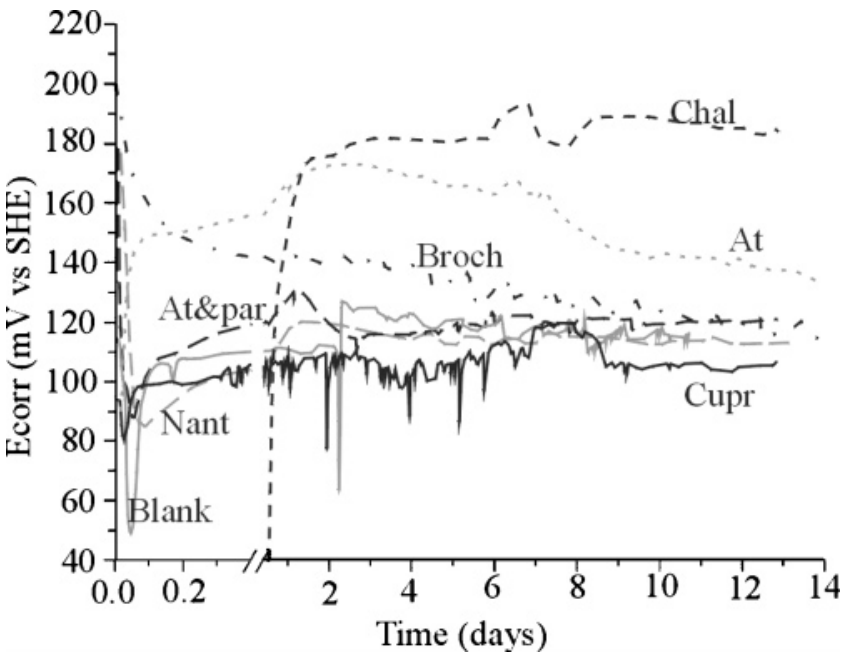

Figure 3. Corrosion potential versus time measurements for a copper-tin-lead alloy covered with different corrosion products and immersed in 1 wt \% sodium sesquicarbonate solution (blank, uncorroded copper-tin-lead alloy; cupr, copper-tin-lead alloy covered with cuprite; nant, copper-tin-lead alloy covered with nantokite; at, copper-tin-lead alloy covered with atacamite; at\&par, copper-tinlead alloy covered with a mixture of atacamite and paratacamite; chal, copper-tin-lead covered with chalcocite; and broch, copper-tinlead alloy covered with brochantite).

corrosion potential is probably caused by the presence of the chalcocite.

The behavior of the copper-tin covered with brochantite resembles the behavior of the copper-tin sample covered with atacamite and chalcocite, except for the start of the immersion. The corrosion potential of the sample covered with brochantite starts to descend right from the beginning of the immersion. The first to second day this descent is rather steep; afterward the slope decreases and becomes the same as is seen with the sample covered with atacamite and chalcocite. SR-XRD data (Table 2) do not show any change in the qualitative composition of the samples before and after immersion; that is, both cuprite and brochantite remain present. The beginning of the corrosion potential versus time plot is obviously affected by the presence of the brochantite. The slow descent of the corrosion potential after these days probably indicates a slight growing of the cuprite layer.

Copper-Tin-Lead. In the same way as for the copper-tin lead samples, a set of artificially corroded copper-tin-lead coupons was prepared according to the above-mentioned corrosion protocols. Also here the obtained corrosion products were verified using SR-XRD and showed that, in contrast to the corrosion of pure copper, where only the desired corrosion product was obtained, ${ }^{10}$ several side products were formed. An overview is given in Table 2.

The corroded coupons were subsequently immersed in a $1 \mathrm{wt}$ $\%$ sodium sesquicarbonate solution while corrosion potential measurements were performed. Measurements were performed at least three times. In Figure 3, a set of representative $E_{\text {corr }}$ measurements for the various corrosion products is shown for an immersion period of 14 days. Comparing the obtained data with the ones plotted in Figure 2, it can be seen at first sight that the corrosion potential of the pure copper-tin-lead alloy is less stable than the one measured for the copper-tin alloy: small to large fluctuations of the corrosion potential are seen for most of 
the samples. This behavior seems to resemble the potential fluctuations noticed during passive film breakdown and repair on iron by Hashimoto and by Isaacs, ${ }^{20-21}$ where the corrosion potential prepitting transients consist of some sharp potential falls followed by a slower return to the stationary value. In the case of the copper-tin-lead alloy, however, these transients can also be due to the heterogeneous structure of the alloy. The metallic $\alpha$-phase consists mainly of copper (88\%) and tin (11\%). Elongated tin powder grains form the grain boundary in the $\alpha$-phase, while lead occurs in large inclusions of pure globules. ${ }^{13}$ Lead possesses a lower potential in the galvanic series in comparison to the copper-tin $\alpha$-phase. The latter implies that, when a pure lead globule comes in contact with the solution, the corrosion potential of the entire surface will adopt a potential between the potential of the pure lead and the potential of the rest of the surface, which explains the potential drops in the corrosion potential. However, since the lead globules are forced to a higher potential by this action, the lead globule corrodes at an accelerated rate. When the complete lead globule has been corroded, or when the corrosion layer formed on top of the globule is protective enough, the corrosion potential will increase again to the value of the copper-tin $\alpha$-phase covered with corrosion products. This phenomenon repeats itself when a new lead globule reaches the surfaces or when small cracks in the corrosion layer permit new contact between a lead globule and the solution. All of the samples showed these fluctuations; however, some are more pronounced than others, which is most probably due to different corrosion layer thicknesses.

In what follows, a more detailed explanation will be given for each of the measurements, while the data will also be linked to the SR-XRD measurements.

Except for the above-mentioned fluctuations in potential, the uncorroded copper-tin-lead alloy and the copper-tin-lead alloy covered with cuprite show a rather stable value within less than $3 \mathrm{~h}$ of immersion. SR-XRD data, however, show the formation of lead carbonates on the cuprite sample (Table 2), which indicates that this compound does not affect the behavior of the corrosion potential as a function of time.

The corrosion potential of the copper-tin-lead alloy covered with nantokite shows a strong decrease at the start of the immersion. After $1.5 \mathrm{~h}$, the corrosion potential increases again and goes through a maximum after $\sim 24 \mathrm{~h}$. The corrosion potential stabilizes after about 3 or 4 days and reaches the same value as the corrosion potential of the uncorroded sample and the one covered with cuprite. SR-XRD spectra taken after 1 day ${ }^{19}$ indicate that during the first day all nantokite is transformed with the formation of cuprite. After 14 days (Table 2), only cuprite is found. The presence of only cuprite is in accordance with the observation that the corrosion potential after 4 days resembles the behavior of the corrosion potential of the copper-tin-lead alloy corroded by the protocol to form pure cuprite.

In the case of the copper-tin-lead sample covered with atacamite, the corrosion potential increases first to a maximum, where it stays stable for a couple of days. Later on, the corrosion potential shows a long slow descent. SR-XRD data (Table 2) show the formation of cuprite and cassiterite. In some cases, malachite

(20) Hashimoto, M.; Miyajima, S.; Murata, T. Corros. Sci. 1992, 33, 885-904. (21) Isaacs, H. I. Corros. Sc. 1993, 34, 525-528. and cerrusite are formed as well. The general behavior resembles that of the copper-tin-lead sample covered with cuprite; however, the influence of the atacamite is reflected in the higher potential of the curve. The corrosion potential seems to evolve slowly to the corrosion potential of copper-tin-lead covered with cuprite, which raises the question of whether atacamite disappears as a function of time. The protocol used to form atacamite produces a loose powdery corrosion layer, and atacamite could possibly be lost due to bad adhesion to the copper surface.

Similar to the case of copper-tin-lead covered with nantokite, the copper-tin-lead covered with the mixture of atacamite and paratacamite shows a strong descending corrosion potential at the start of the immersion. After $1.5 \mathrm{~h}$, the corrosion potential increases and goes through a maximum after a little more than 1 day. The corrosion potential stabilizes after $\sim 2.5$ days around the same value as the corrosion potential of the uncorroded coppertin-lead and the copper-tin-lead covered with cuprite. SR-XRD data (Table 2) show the formation of cuprite, cassiterite $\left(\mathrm{SnO}_{2}\right)$, massicot $(\mathrm{PbO})$, and hydrocerrussite $\left(\mathrm{Pb}_{3}\left(\mathrm{CO}_{3}\right)_{2}(\mathrm{OH})_{2}\right)$ during the immersion. On some of the samples, malachite is formed as well. The cuprite seems to have a stabilizing influence on the behavior of the corrosion potential. Similar to the case of copper-tin-lead covered with nantokite, the corrosion potential of the coppertin-lead covered with the mixture of atacamite and paratacamite resembles the behavior of the copper-tin-lead covered with cuprite after 4 days immersion. The influence of the other corrosion products (cassiterite, massicot, hydrocerrusite) is not clear at this stage. Presumably these products are too low in concentration to have an influence on the corrosion potential and probably also to have an influence on the corrosion behavior.

The corrosion potential versus time plot of copper-tin-lead covered with brochantite starts with a rather strong decrease, followed by a less steep descent, and stabilizes around the value of the corrosion potential of the bare copper-tin-lead alloy. This is the same behavior as the copper-tin alloy covered with brochantite, and also in this case, the SR-XRD results show cuprite was already present before the start of the immersion. The SR-XRD results also show the formation of cerrusite $\left(\mathrm{PbCO}_{3}\right)$, but apparently the formation of this product does not influence the behavior of the corrosion potential in function of time.

The copper-tin-lead covered with chalcocite shows a completely different behavior. The first day, the corrosion potential shows a very strong increase. Afterward a more or less stable value is obtained, but at a higher potential than the rest of the samples. SR-XRD results show the formation of cassiterite $\left(\mathrm{SnO}_{2}\right)$, litharge $(\mathrm{PbO})$, cerrusite $\left(\mathrm{PbCO}_{3}\right)$, and hydrocerrusite $\left(\mathrm{Pb}_{3}\left(\mathrm{CO}_{3}\right)_{2-}\right.$ $(\mathrm{OH})_{2}$ ), which are most probably the cause of the specific behavior of the corrosion potential. For the moment, it is not known how the corrosion potential is influenced by each of the components. New tests with these corrosion products need to be performed to sort this out.

\section{DISCUSSION}

The reactions of the different samples seem to depend mostly on the corrosion product covering the sample and less on the alloy substrate. Four types of behaviors during the 14 days of immersion can be distinguished (Figure 4):

Type 1: The corrosion potential shows a steep rise at the beginning of the immersion, which ends in a more or less stable 


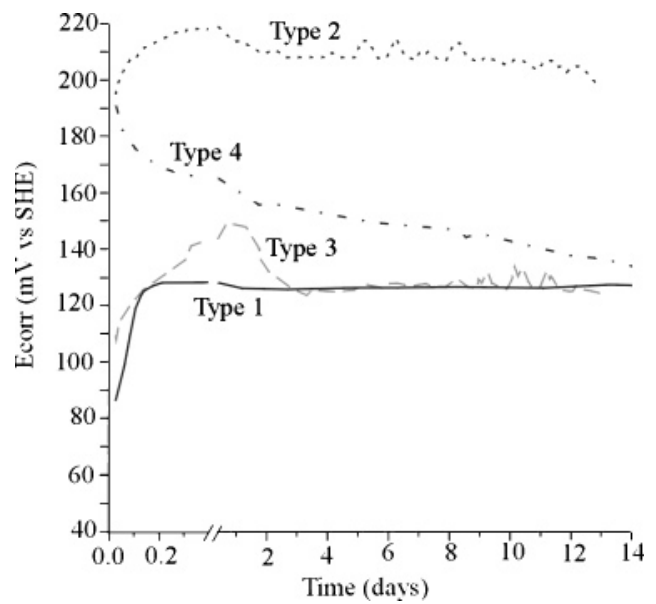

Figure 4. Four typical corrosion potential versus time measurements for a copper-based samples covered with different corrosion products immersed in $1 \mathrm{wt} \%$ sodium sesquicarbonate.

potential after only a few hours. This behavior can be seen in the case of uncorroded samples and samples covered with cuprite. SR-XRD measurements of the surfaces do not show any change before or after treatment.

Type 2: Atacamite and chalcocite show a behavior similar to type 1 , but the potentials are higher and the "stable" potential slowly descends. SR-XRD spectra show the formation of cuprite and sometimes other corrosion products such as malachite, cassiterite, and lead carbonates in the case of copper-tin-lead alloy.

Type 3: The corrosion potential of the samples covered with nantokite and with a mixture of paratacamite and atacamite goes through a maximum after 1-4 days. Afterward, the potential progresses to the corrosion potential of samples covered with cuprite. SR-XRD spectra show that nantokite and paratacamite convert into cuprite and malachite, which is confirmed in the literature. ${ }^{4-6}$ The transformation of nantokite, and at slower rate of paratacamite, seems to take place during the first descent and the increase of the corrosion potential. Tests on copper samples covered with nantokite revealed that the maximum of the corrosion potential versus time plot coincided with the transformation of the last nantokite present on the sample. After the maximum of the corrosion potential, only cuprite is found on the sample. ${ }^{11}$ In a later stage, malachite formed as well. ${ }^{10}$

Type 4: The last type of corrosion potential curve only occurs for the samples covered with brochantite. First there is a rather strong descent followed by a more steady descent, the latter resembling the behavior of the samples covered with chalcocite and with atacamite. SR-XRD spectra do not show any difference before and after 14 days immersion in the sodium sesquicarbonate solution.

By comparing the reactions of the alloys with the reaction of pure copper covered with the same corrosion products, ${ }^{10}$ it can be seen that, in general, the corrosion potential of the alloys shows variations with time similar to the pure copper. However, a few differences must be noted.

For the alloys covered with nantokite, the corrosion potential starts with a small increase after 13 days of immersion (Figure 4 , type 3); this increase starts earlier for the copper samples covered with nantokite (after 6-7 days). ${ }^{10}$ The latter is attributed to the formation of malachite. This seems to indicate that the formation of malachite is easier on copper than on the alloys.

Also, for the samples covered with atacamite and chalcocite, there is a small difference between the alloys and pure copper. For alloys covered with atacamite as well as for copper covered with chalcocite, the corrosion potential evolves into a descent after 2-3 days of immersion (Figure 4, type 2). For copper covered with these corrosion products, however, the corrosion potential increases slowly instead of evolving into a descent. ${ }^{10}$ Only by the end of the 14 days of immersion, the corrosion potential finally seems to evolve to a stable corrosion potential. XRD spectra of the different samples show that cassiterite is formed on the two alloys during the immersion. This product did not occur on the copper sample and can be a possible cause for the different behavior.

\section{CONCLUSIONS}

This work has contributed to the research question whether corrosion potential measurements can be used as an early warning system to monitor the behavior of copper-based artifacts during their storage and stabilization treatments. The hypothesis behind the method is that, if the corrosion potential does not change as a function of time, the surface composition of the corroded alloy should be stable. A change of the corrosion potential, on the other hand, is to indicate a transformation of the natural patina or the development of active corrosion.

In this study, two alloys, copper-tin and copper-tin-lead, were artificially corroded with the most common corrosion products observed on copper alloys. The prepared samples were immersed in a $1 \%$ sodium sesquicarbonate solution during a period of 14 days, while corrosion potential measurements were performed. SR-XRD measurements were performed on the sample surface before and after the treatment to evaluate the potential change in surface composition.

Results show that the behavior of the corrosion potential as a function of time is clearly different for the various corrosion products examined, while the influence of the substrate seems less important. The composition of the substrate, however, does have an influence on the actual value of the corrosion potential. The corrosion potential of pure copper covered or not with the corrosion products is $\sim 15 \mathrm{mV}$ more positive than that for the copper-tin alloy covered with the same corrosion products, which in its turn lies $\sim 20 \mathrm{mV}$ less negative than the corrosion potential for the copper-tin-lead alloy covered with the same corrosion products.

On the other hand, variations of the corrosion potential as a function of time mainly depend on the composition of the corrosion layer present. Changes of the corrosion potential therefore reflect compositional changes taking place at the surface. Especially the transformation of the aggressive copper chloride species seems well reflected in the behavior of the corrosion potential.

We are currently examining the possibility of simultaneous SR-XRD and electrochemical measurements. The benefits of in situ analysis are the avoidance of air and vacuum exposure with consequently unknown surface changes and the minimization of the number of samples required. The latter point is especially important because it means that the method can be used on the restricted numbers of real artifact fragments that can be released for testing. First results on copper using a specially designed 
electrochemical cell have been published. ${ }^{11}$ We intend to expand this work to copper alloys. Moreover, additional experiments are planned with multilayer patinas to further evaluate the monitoring method.
Received for review December 12, 2005. Accepted February 1, 2006.

AC052192S 